PACS 85.60.Jb

\title{
White LED modules encapsulation features
}

\author{
Yu.V. Trofimov, V.S. Posedko, E.F. Ostretsov, V.I. Tsvirko, L.N. Survilo, N.N. Marus \\ Center of LED and Optoelectronic Technologies of National Academy of Sciences of Belarus, \\ Logoisky trakt 22, 220090 Minsk, Belarus \\ Phonelfax: +375(017)-281-13-62, e-mail: senso@inel.bas-net.by
}

\begin{abstract}
The behaviour and causes of changes for correlated color temperature (CCT) and radiant spectral power in white LED modules of different design at their encapsulation by optical compounds are investigated. It is shown that CCT of original white LED radiation can both decrease and increase after polymeric covering application. The CCT variation direction is determined mainly by primary lens presence on LED. The variation value of color parameters of white LED modules is determined by created optical surface curvature and spectral characteristics of deposited polymer.
\end{abstract}

Keywords: LED, encapsulation, color temperature.

Manuscript received 11.12.12; revised version received 24.01.13; accepted for publication 19.03.13; published online 25.06.13.

\section{Introduction}

One way of LED modules protection against negative environment impact and vandalism is their encapsulation by special optically transparent compounds [1]. In practice, it provides the effective moisture protection, vibration and shock resistance of LED modules, allows to exclude the air spaces between LED module and protective glass, to reduce the losses at optical medium boundaries, as well as to reject the protective glass in certain cases.

However, the compound application directly on LED produces inevitable changes of optical parameters of resultant light radiation [2]. First, applied polymer layer changes the configuration of LED optical system and radiation angular distribution. Second, the polymer serves as an additional light filter reducing the total light flux and changing colour parameters in accordance with the spectral transmission characteristic of used polymer.

\section{Experimental results and discussion}

LED and polymer compound light characteristics were measured with scanning spectrophotometer SPECTRO 320 and goniophotometer LEDGON 100 (Instrument Systems). All measured LEDs were based on white luminophor.

Under white LED encapsulation, the change of correlated color temperature and other colour parameters is occurred. The radiation chromaticity changing the mechanism of white luminophor LEDs is provided by radiation redistribution between blue and yellow-green spectral regions (Fig. 1).

So, filling of LED without primary lens, i.e. with flat or slightly concaved optical surface, results in formation of the convex or flat surface. It, in its turn, increases the part of output light radiation in blue spectral region $\mathbf{B}$ and decreases the luminophortransformed part of radiation $\mathbf{Y}$. As a result, CCT of the total light flux $\mathbf{W}$ is shifted to cooler region. 

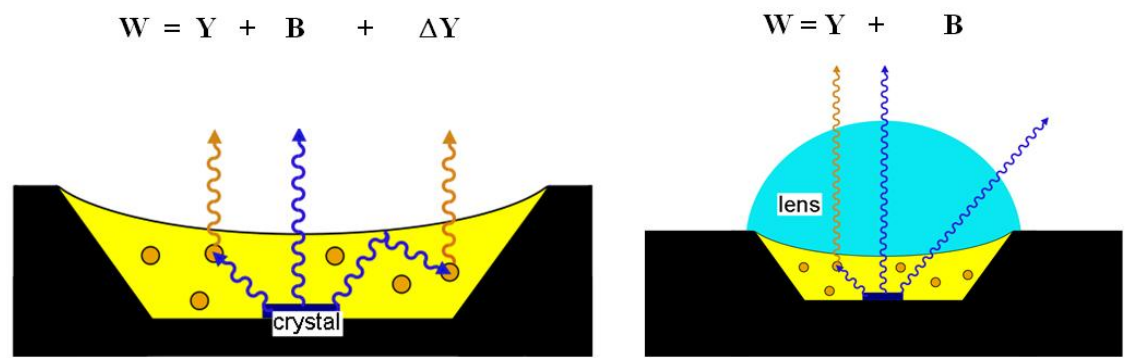

a)
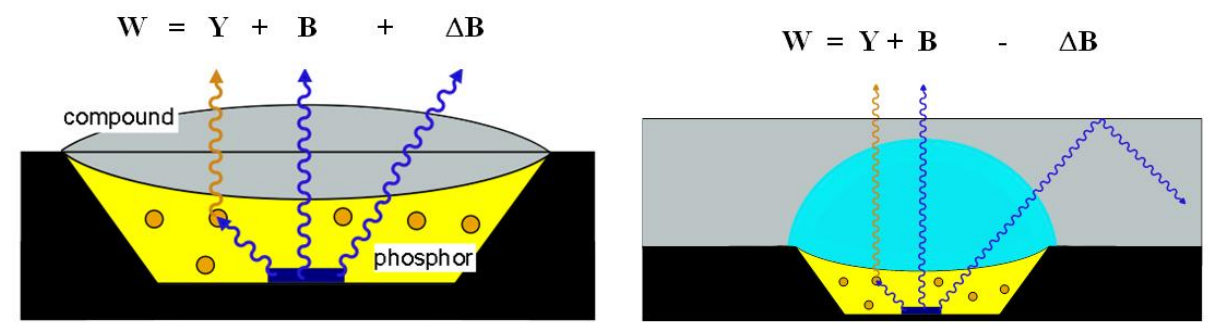

b)

Fig. 1. Simplified comparative model of radiation coupling for LED with and without lens before (a) and after (b) compound application.

The radiant spectral power change of NS6W083A (Nichia) LED after silicon lens formation is shown in Fig. 2.

Essential LED optical surface curvature change at the lens-air boundary results in CCT averaged value growth from 5240 up to $9060 \mathrm{~K}$.

Layer-to-layer application of compound with uniform transmission characteristic in white LED radiation range on LED without lens results in a monotonous increase of the blue component in the total light flux and respective CCT growth.

The radiant spectral power density change and CCT for NS6W083A LED with layer-to-layer acrylic compound PLASTIK (Cramolin, Germany) application are shown in Fig. 3.

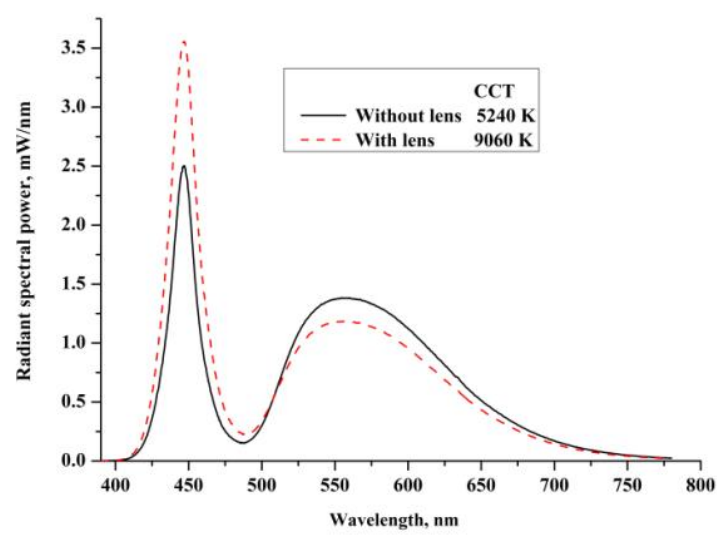

Fig. 2. The NS6W083A LED radiant spectral power dependence on the wavelength before and after silicon lens formation.
Compound is applied by pulverization and gets airdried at room temperature. The thickness of single layer is equal to $25 \ldots 30 \mu \mathrm{m}$. The compound uniform transmission characteristic practically excluded its influence on colour parameters as an additional light filter.

With layer-to-layer application of polyurethane compound URETHANE (Cramolin, Germany) with the non-uniform transmission characteristic, its influence as additional light filter is evident only for two layers, not essentially changing the optical system curvature (Fig. 4b).

This becomes apparent in initial decrease of the blue component fraction in the total light flux and, as a consequence, CCT. At further layers application, when the compound - air boundary curvature is changed from concave to flat or even convex form, the blue component fraction in the total light flux and CCT are increased again.

It is confirmed in practice at LED lamp production when LED without primary lens has a contact with transparent compound. The light color is turned into cool white, and its temperature is changed, for example from 5000 up to $8000 \mathrm{~K}$.

At compound application of LEDs with primary lens, another dynamics of chromaticity changing is observed. In this case, the optical system surface curvature is changed from convex to flat or even to concave form (Fig. 1). The blue component fraction in the total light flux is decreased, and CCT is shifted to a warmer region.

The dependences of radiant spectral power density change for cool white (NFSW036L, Nichia) (a) and warm white (NFSL036L, Nichia) (b) LEDs with 
acrylic compound encapsulation on wavelength are shown in Fig. 5. The corresponding CCT values are indicated inside the figure. The filling is prolonged until flat surface adjoining with air is formed.
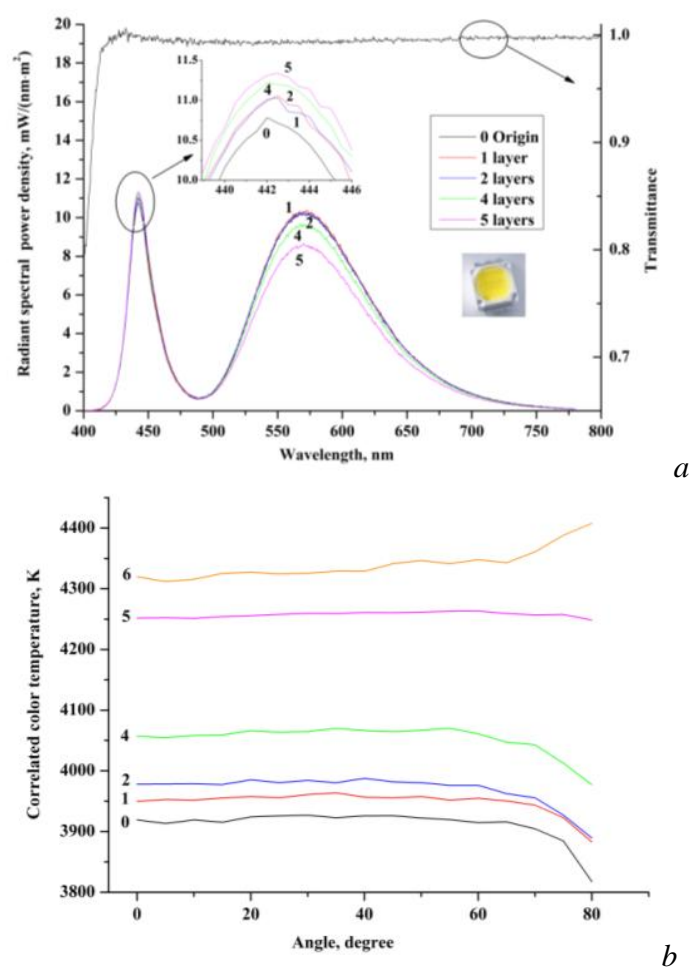

Fig. 3. The radiant spectral power density change (a) and CCT (b) for NS6W083A LED with layer-to-layer applied acrylic compound.
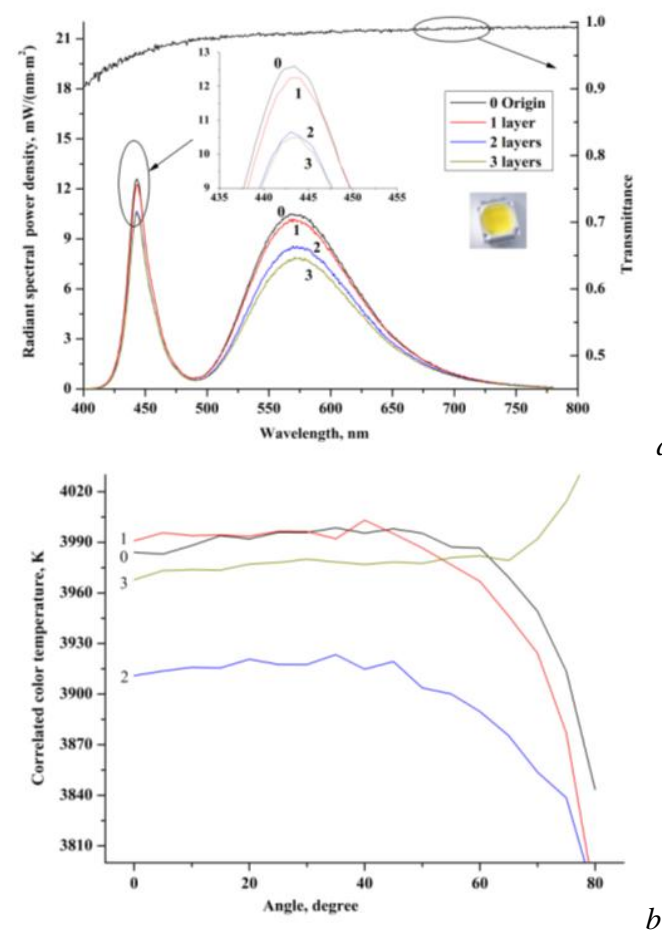

Fig. 4. The radiant spectral power density (a) and CCT (b) change for NS6W083A LED with layer-to-layer applied polyurethane compound.
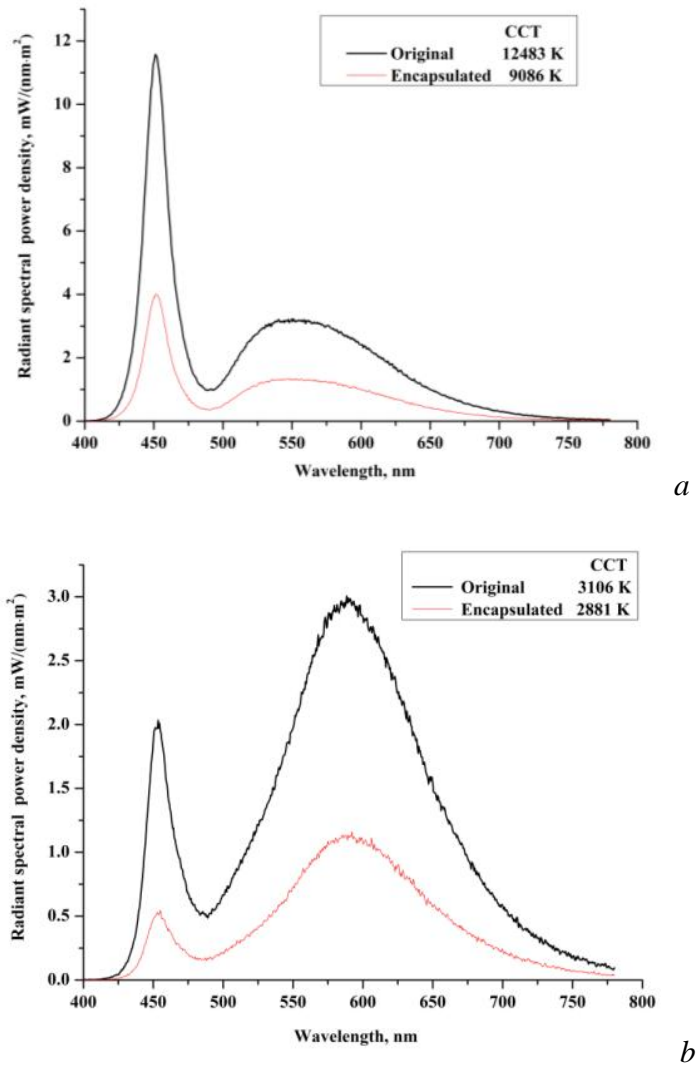

Fig. 5. The dependence of radiant spectral power density change for cool white (a) and warm white (b) LEDs on the wavelength before and after acrylic coating formation.

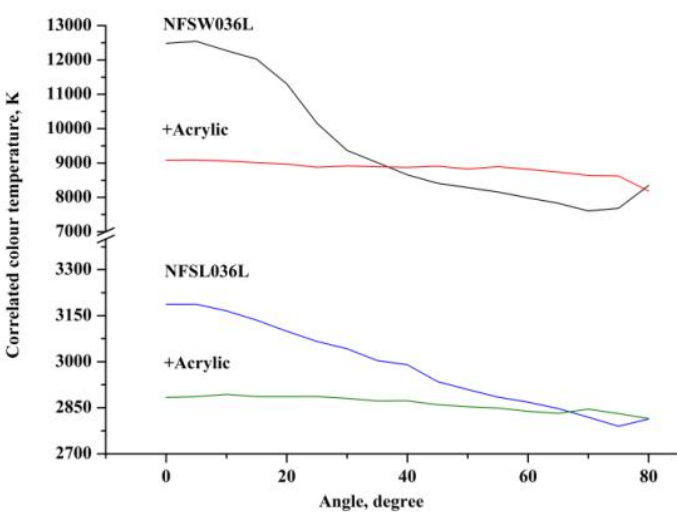

Fig. 6. CCT angular distribution for cool white NFSW036L and warm white NFSL036L LEDs before and after acrylic coating formation.

The correlated color temperature for cool white LEDs $(\mathrm{CCT}=12483 \mathrm{~K})$ was decreased by $27 \%$, and for warm white LEDs $(\mathrm{CCT}=3106 \mathrm{~K})-$ by $7 \%$, respectively. As seen from Fig. 6, CCT angular distribution becomes more uniform.

The carried out investigations are shown that compound type does not affect much on both CCT change and CCT angular distribution. 


\section{Conclusion}

At white LED modules encapsulation by compound application, the significant CCT change takes place. The basic reason for this is the change of LED optical system curvature at the boundary with air.

The modification tendency is defined by the primary lens presence or absence: when it is present, the CCT is decreased, when it is absent, the CCT is enhanced.

The possible ways for avoiding the changing of color parameters are as follows:

- sharing LEDs with and without lens for CCT cancellation after filling;

- encapsulation technology development with a small air gaps under LED formation.

\section{References}

1. A. Petrov, Let be light! Modern LED lighting. Trends. Objectives. Solutions // Sovremennaya svetotekhnika, 2(09), p. 43-46 (2011), in Russian.

2. Yu.V. Trofimov, V.S. Posedko, E.F. Ostretsov, V.I. Tsvirko, L.N. Survilo, Influence research of waterproof coatings on lighting parameters of LEDs // 8-th Belarusian-Russian Workshop "Semiconductor lasers and systems", p. 206-209 (2011). 